\title{
Evolutionary and functional implications of hypervariable loci within the skin virome
} \author{
Elizabeth A Grice ${ }^{\text {Corresp. } 1,3}$ \\ ${ }^{1}$ Dermatology, University of Pennsylvania, Philadelphia, PA, United States \\ 2 One Codex, San Francisco, CA, United States \\ 3 Microbiology, University of Pennsylvania, Philadelphia, PA, United States \\ Corresponding Author: Elizabeth A Grice \\ Email address: egrice@upenn.edu
}

Geoffrey D Hannigan ${ }^{1}$ ， Qi Zheng ${ }^{1}$ ， Jacquelyn S Meisel ${ }^{1}$, Samuel S Minot ${ }^{2}$, Frederick D Bushman ${ }^{3}$,

Localized genomic variability is crucial for the ongoing conflicts between infectious microbes and their hosts. An understanding of evolutionary and adaptive patterns associated with genomic variability will help guide development of vaccines and antimicrobial agents. While most analyses of the human microbiome have focused on taxonomic classification and gene annotation, we investigated genomic variation of skinassociated viral communities. We evaluated patterns of viral genomic variation across 16 healthy human volunteers. HPV and Staphylococcus phages contained 106 and 465 regions of diversification, or hypervariable loci, respectively. Propionibacterium phage genomes were minimally divergent and contained no hypervariable loci. Genes containing hypervariable loci were involved in functions including host tropism and immune evasion. HPV and Staphylococcus phage hypervariable loci were associated with purifying selection. Amino acid substitution patterns were virus dependent, as were predictions of their phenotypic effects. We identified diversity generating retroelements as one likely mechanism driving hypervariability. We validated these findings in an independently collected skin metagenomic sequence dataset, suggesting that these features of skin virome genomic variability are widespread. Our results highlight the genomic variation landscape of the skin virome and provide a foundation for better understanding community viral evolution and the functional implications of genomic diversification of skin viruses. 
1
Title

Evolutionary and Functional Implications of Hypervariable Loci Within the Skin Virome

\section{Authors}

Geoffrey D. Hannigan ${ }^{1}$, Qi Zheng ${ }^{1}$, Jacquelyn S. Meisel ${ }^{1}$, Samuel Minot ${ }^{2}$, Frederick D. Bushman ${ }^{3}$, Elizabeth A. Grice*1,3

\section{Author Affiliation}

${ }^{1}$ Department of Dermatology, University of Pennsylvania Perelman School of Medicine; ${ }^{2}$ One Codex, San Francisco, CA; ${ }^{3}$ Department of Microbiology, University of Pennsylvania Perelman School of Medicine

\author{
${ }^{*}$ Correspondence \\ Elizabeth A. Grice, $\mathrm{PhD}$ \\ Department of Dermatology and Microbiology \\ Perelman School of Medicine \\ University of Pennsylvania \\ 421 Curie Blvd, 1007 BRB II/III \\ Philadelphia, PA 19104 \\ 215-898-3179 (phone) \\ 215-573-2033 (fax) \\ egrice@upenn.edu
}




\section{ABSTRACT}

26 Localized genomic variability is crucial for the ongoing conflicts between infectious microbes and their

27 hosts. An understanding of evolutionary and adaptive patterns associated with genomic variability will

28 help guide development of vaccines and anti-microbial agents. While most analyses of the human

29 microbiome have focused on taxonomic classification and gene annotation, we investigated genomic

30 variation of skin-associated viral communities. We evaluated patterns of viral genomic variation across 16

31 healthy human volunteers. HPV and Staphylococcus phages contained 106 and 465 regions of

32 diversification, or hypervariable loci, respectively. Propionibacterium phage genomes were minimally

33 divergent and contained no hypervariable loci. Genes containing hypervariable loci were involved in

34 functions including host tropism and immune evasion. HPV and Staphylococcus phage hypervariable loci

35 were associated with purifying selection. Amino acid substitution patterns were virus dependent, as were

36 predictions of their phenotypic effects. We identified diversity generating retroelements as one likely

37 mechanism driving hypervariability. We validated these findings in an independently collected skin

38 metagenomic sequence dataset, suggesting that these features of skin virome genomic variability are

39 widespread. Our results highlight the genomic variation landscape of the skin virome and provide a

40 foundation for better understanding community viral evolution and the functional implications of genomic

41 diversification of skin viruses. 


\section{INTRODUCTION}

44 Localized genomic modifications are ammunition in the ongoing battle between hosts and infectious 45 agents. The human adaptive immune response relies on localized genomic diversity of antigen receptors 46 to facilitate detection and efficient removal of foreign agents (Borghans, Beltman \& De Boer, 2004; 47 Kubinak et al., 2012). Infectious microbes, such as bacteria and viruses, likewise rely on genomic 48 variation to modulate tropism and facilitate immune evasion (e.g. (Malim \& Emerman, 2001; Doulatov et 49 al., 2004; Minot et al., 2012; Schillinger et al., 2012; Das et al., 2013; Minot et al., 2013; Guo et al., 50 2014)). Potential selective benefits of targeted variation in viruses include immune evasion and widening 51 of host tropism (Borghans, Beltman \& De Boer, 2004; Kubinak et al., 2012).

Most contemporary low resolution studies of the human microbiome evaluate functional potential through taxonomic classification and whole gene identification (e.g. (Schloss \& Handelsman, 2008; Human Microbiome Project Consortium, 2012; Langille et al., 2013; Hannigan et al., 2014; Ly et al., 2014; Norman et al., 2015; Lim et al., 2015; Meisel et al., 2016)). These approaches are usually unable to capture nucleotide variations that affect functionality of proteins encoded in the microbiome, which can be altered by differences in only a few nucleotides. For example, viruses such as Bordetella bacteriophages, hepatitis $\mathrm{C}$ virus, and others only require short variable regions within a gene to facilitate functional changes in processes including tropism diversity, immune evasion, drug resistance, and adaptation to host auxotrophies (Bacher, Bull \& Ellington, 2003; Doulatov et al., 2004; Donaldson et al., 2010; Guan et al., 2012; Shah et al., 2014). Contemporary low-resolution studies also fail to identify genetic cassettes that promote targeted diversity, such as diversity generating retroelements (DGRs). DGRs promote targeted genetic diversification in bacteriophages through error-prone cycles of transcription, reverse transcription, and integration; through this process information encoded in a nonvariable template region is copied in a fallible fashion into a variable region within a coding sequence

67 (Doulatov et al., 2004; Minot et al., 2012; Schillinger et al., 2012). 
69 Here we investigate skin virome evolution and adaptation by inferring the selective pressure, functional

70 diversity, and substitution patterns associated with targeted hypervariation. We focus on three prominent

71 cutaneous viruses: Human Papillomavirus (HPV), Propionibacterium phage, and Staphylococcus phage.

72 HPV is associated with the development of skin cancer, especially in immune-suppressed individuals

73 (Vinzón et al., 2014; Wang et al., 2014; Quint et al., 2015). Current vaccine efforts aim to target

74 conserved antigens for broad strain protection - thus a greater understanding of HPV genomic diversity

75 could improve design of vaccines (Schiller \& Lowy, 2012; Vinzón et al., 2014). Staphylococcus phages

76 can modulate Staphylococcus pathogenic gene expression and facilitate transmission of antibiotic

77 resistance (Bae et al., 2006; Varga et al., 2012). Propionibacterium phages are associated with

78 Propionibacterium acnes and have therapeutic potential for treating acne (Marinelli et al., 2012;

79 Hannigan \& Grice, 2013). Our findings build upon previous analyses of individual virus genomic

80 variability and provide new insight into phage biology of the cutaneous microbiome.

81

82

MATERIALS \& METHODS

Analysis Details and Availability

84 All associated source code and explanatory README files are available for review at the following

85 GitHub repository: https://github.com/Microbiology/ViromeVarScripts.

86

87 Data Acquisition \& Quality Control

88 The primary skin virome dataset was acquired from SRA accession: SRP049645 (Hannigan et al., 2015)

89 and includes sequences from samples collected at the second and third time points. The secondary dataset

90 was obtained from Oh et al (SRA BioProject: 46333) (Oh et al., 2014). Retroauricular crease samples

91 were downloaded from the NCBI SRA BioProject: 46333. For samples from both the primary and

92 secondary dataset, sequences were trimmed with the FASTX-Toolkit (version 0.0.14), using a quality

93 score cutoff of 33 . Remaining sequences with similarity to the human genome were removed using the

94 standalone DeconSeq toolkit (version 0.4.3) (Schmieder \& Edwards, 2011). 
96 Contig Assembly and Taxonomic Identification

97 Contigs from the primary dataset were obtained from the published Figshare source (doi:

98 10.6084/m9.figshare.1281248). Contigs from both the primary and secondary datasets were separately

99 assembled using the Ray metagenomic assembly software, specifying a minimum contig length of 500bp

100 and otherwise default parameters (v2.3.1) (Boisvert et al., 2012). Within each dataset, sequences from all

101 samples were combined prior to assembly to facilitate the most complete contig assembly. Contig

102 coverage was determined by aligning sequences back to the contigs with the bowtie2 toolkit (v2.1.0; seed

103 substring length of 25 and one mismatch allowed in alignment) (Langmead \& Salzberg, 2012).

104 Quantification of reads mapping back to contigs was obtained by parsing bowtie2 output using Perl and

105 BASH scripts presented in the supplemental source code. Coverage was calculated using the number of

106 reads mapping to each contig. The blastn program from the NCBI Blast + toolkit (version 2.2.0) was used

107 to determine similarity of contigs to virus reference genomes (Camacho et al., 2009). Contigs were

108 blasted against a previously described virus-specific genome reference database, which is a subset of the

109 EMBL reference genome database (UniProt Consortium, 2014; Hannigan et al., 2015). A similarity

110 threshold of e-value $<10^{-3}$ was used, and sequences with multiple potential identities were resolved by

111 using only hits with the lowest e-values. Although this was the minimum threshold, the contigs of interest

112 exhibited e-values much lower than $10^{-3}$.

114 Phylogenetic Analysis

115 We constructed phylogenies using the L1 capsid gene for HPV (Ma et al., 2014) and the large terminase 116 subunit for the Staphylococcus and Propionibacterium phages (Gutiérrez et al., 2013; Ma et al., 2014) as 117 phylogenetic marker genes. For reference, we used the PAVE reference L1 genes (<pave.niaid.nih.gov>, 118 accessed 2015-06-03) (Van Doorslaer et al., 2013). The large terminase subunit references for 119 Staphylococcus and Propionibacterium phages were from the NCBI gene sequence database 120 (Staphylococcus phage: accessed 2015-09-14, search term: ((phage terminase large subunit 
121

122

123

124

125

126

127

128

129

130

131

132

133

134

135

136

137

138

139

140

141

142 NCBI nucleotide database using the search term ""Staphylococcus"[Organism] AND "complete

143 genome"[Name] NOT phage[All Fields] NOT contig[All Fields] NOT ("unidentified plasmid"[Organism]

144 OR plasmid[All Fields]) AND (bacteria[filter] AND biomol_genomic[PROP])". Both were accessed

145 December 22, 2016. Together this allowed us to detect regions of contigs that demonstrated a high

146 similarity to temperate phage gene signatures. 
148 Identification of Hypervariable Loci

149 The bowtie2 alignments of reads to viral contigs were formatted (e.g. conversion from binary to ASCII

150 format) and then SNPs were called using VarScan (v2.3.7) (Li et al., 2009; Koboldt et al., 2012). The

151 'pileup2snp' program from VarScan was used with a minimum minor allele frequency threshold of 1\%, a

152 read depth of 8, and a minimum of two supporting reads for variant calls. Indels were excluded.

153

154 To identify hypervariable loci, we used a geometric distribution based statistic approach as described 155 previously (Zheng et al., 2010), which, compared to sliding window searches and other similar methods, 156 has the advantage of avoiding boundary difficulties and variations within contigs. We used a geometric 157 distribution to model the probability of achieving two SNPs separated by a specified non-SNP nucleotide 158 distance. Each between-SNP distance was associated with a probability and the probability of a particular 159 distance occurring by randomly sampling was less than 5\%. Thus, we identified a range of SNP distances 160 as significantly less than background if they occured within our dataset less than $5 \%$ of the time.

161

162

Protein Family Domain Identification Within Hypervariable Loci ORFs

163

Protein family domains were identified in ORFs that contained hypervariable loci. The subset of translated virus ORFs that contained hypervariable loci were aligned to the standard Pfam protein family 165 domain database using hmmscan within the HMMer toolkit (version 3.1) and GA gathering bit score thresholds (Finn, Clements \& Eddy, 2011).

167

\section{Prediction of Single Amino Acid Variant Effect on Phenotype}

169 The SuSPect algorithm was employed to predict the likelihood of SNP-associated single amino acid 170 variants (SAV) impacting phenotype. We used SuSPect to create a matrix of likelihood scores for every 171 possible SAV at every position in the ORFs that contained hypervariable loci. This matrix was used as a 
172 reference to quantify the likelihood of each hypervariable loci SNP to impact the resulting phenotype. The

173 significance of the score differences between viruses was calculated using a Wilcoxon rank-sum test.

174

175 Evolutionary Pressure of Hypervariable Loci and Virus Genomes

176 We assessed the evolutionary pressure of a gene using the $p \boldsymbol{N} / \boldsymbol{p} S$ ratio as in Formula 1, where $\mathrm{M}_{\mathrm{N}}$ and

$177 \mathrm{M}_{\mathrm{S}}$ represent the observed number of non-synonymous and synonymous SNPs, respectively. These values

178 were normalized by the total number of possible non-synonymous or synonymous substitutions $\left(\mathrm{N}_{\mathrm{i}}\right.$ and $\mathrm{S}_{\mathrm{i}}$,

179 respectively), in order to avoid potential codon usage bias. Furthermore, to normalize for sequence

180 coverage of the SNPs and prevent extreme values, a pseudocount value of an arbitrarily small number

181 was added to the $\mathrm{M}_{\mathrm{N}}$ and $\mathrm{M}_{\mathrm{S}}$ values, which was defined as half of the square root of the median sequence

182 coverage of SNPs within the region of interest $\left(\mathrm{C}_{M}\right)$. The pseudocount approach was used to prevent

183 infinite and illegal values when $\mathrm{M}_{\mathrm{N}}$ or $\mathrm{M}_{\mathrm{S}}$ had zero values, thus allowing consideration of otherwise

184 infinite or ignored data points. For example, an absence of synonymous mutations would result in an

185 infinitely large value (dividing by zero) thus forcing exclusion of the data point. Our approach preserves

186 this data and allows us to draw conclusions from the largest possible dataset, and has been shown to be

187 effective in previous studies (Novaes et al., 2008; Bajgain et al., 2011).

188

189 This analysis is similar to the $\boldsymbol{d} \boldsymbol{N} / \boldsymbol{d} \boldsymbol{S}$ calculations often performed to estimate degrees of natural selection among genomes (Nishida, Frith \& Nakai, 2009; Schloissnig et al., 2013). It is important to note however that such an analytical approach would be inappropriate for this type of sample set because the nucleotide variations are not assignable to isolated strains, which prevents haplotype identification that is a necessary component of $d N / d S$ calculations. The $p N / p S$ calculation does not assume haplotypes, and is therefore

194 appropriate for metagenomic datasets.

196 To estimate the selective pressure on hypervariable loci, the locations of the hypervariable loci were 197 extracted, along with their immediately adjacent regions, using a Perl script as presented in the 
198 supplemental source code. Adjacent regions are defined as the genomic regions that are two times the

199 length of the hypervariable loci and located immediately before and after the hypervariable loci. These 200 positions were used with the contig sequences, SNP call data, and $p N / p S$ calculator to estimate their 201 selective pressure. Hypervariable regions outside of coding regions were not considered.

202

203 Calculation of the overall selective pressure on virus contigs was performed in a similar approach to the 204 hypervariable loci selective pressure. Predicted ORFs were first extracted from the contigs using the 205 Glimmer3 toolkit (v3.02) (Delcher et al., 2007). The predicted ORFs, along with the contig sequences, 206 SNP profile, and $p N / p S$ calculator were used to calculate the overall selective pressure on each gene 207 within each contig. The distributions of selective pressures observed for each gene were observed as 208 categorized by virus type.

209

210 Amino Acid Frequency, Charge, and Polarity

211 Amino acid abundance profiles were calculated while correcting for the random probability of that 212 substitution. More specifically, each value was weighted for the number of nucleotides that result in the 213 same amino acid as weighted value =((number of nucleotide substitutions resulting in same amino acid) / $2143)^{-1}$. Relative abundance was calculated as the sum of the corrected frequencies. Charge and polarity were 215 determined using a simple table of known amino acid properties. Differences in profiles between viruses 216 were calculated using a chi-square test.

217

218 Diversity Generating Retroelement Identification

219 We identified potential diversity generating retroelements (DGRs) by collecting assembled contigs that 220 contained open reading frames similar to known reverse transcriptase genes, and a duplicated nucleotide 221 region less than $150 \mathrm{bp}$ in length. Reverse transcriptase open reading frames were identified using blastx 222 (e-value $<10^{-5}$ ) and the Uniprot reference reverse transcriptase sequences 223 (http://www.uniprot.org/uniprot/?sort=score\&desc=\&compress=yes 
$224 \&$ query $=\% 22$ reverse $\% 20$ transcriptase $\% 22 \% 20$ (phage $\% 200 R \% 20$ virus $) \&$ fil $=\&$ format $=$ fasta $\&$ force=yes $).$

225 Repeat regions were identified by comparing each contig to itself with tblastx (e-value $<10^{-50}$ ) and were 226 filtered using customt scripts to remove duplicates and regions longer than 150bp. DGR candidates were 227 removed if they contained no hypervariable loci or if the variable region was no within a predicted open 228 reading frame.

229

230 Diversity generating retroelements were visualized in the Integrated Genomic Viewer using the DGR 231 cassettes and bowtie2 aligned sequences described above. The linkage disequilibrium was calculated 232 using a custom perl script for formatting and the "LDheatmap" and "genetics" R packages for analysis 233 and visualization (Shin et al., 2006; Warnes et al.). The linkage disequilibrium for each pair of SNPs was calculated as the squared allelic correlation $\left(\mathrm{R}^{2}\right)$.

235

236

Comparison of Primary Analysis to Validation Dataset

237

Near identical contigs were identified between the primary and secondary validation dataset by aligning the two individually assembled contigs to each other using bowtie2, with a specified seed length of 25 and up to one seed mismatch. Sequences from our primary dataset and the Oh et al dataset were aligned to the near identical contigs. These alignments were used to identify shared SNP locations between our 241 dataset and the Oh et al dataset. We quantified shared SNP location as percent of our primary analysis 242 SNPs whose location was identical to those of SNPs in the secondary validation dataset. As a control, we 243 compared these results to a simulated dataset where SNP position was randomly assigned. The example 244 SNP alignment over the circular contig was generated using Genious (Kearse et al., 2012). 


\section{RESULTS}

\section{Diversity of Skin Viruses}

249 We evaluated genomic variability associated with dsDNA skin viruses using a previously published 250 human skin virome metagenomic dataset, consisting of 260,714,906 high quality sequences assembled 251 into $>$ 76,000 contigs from 16 individuals (SRA Accession: SRP049645) (Hannigan et al., 2015). We 252 relied on database virus annotation to identify the taxonomic groups whose contigs had the overall highest 253 confidence matches to reference genomes. Because greater sequencing coverage allows for more refined 254 detection of variable nucleotides (Schloissnig et al., 2013), we focused our analysis on taxa whose de 255 novo assembled contigs had sufficient coverage (greater than 10X). Contigs meeting these criteria were 256 identified as Propionibacterium phages (contig count $=45)$, Staphylococcus phages $($ contig count $=319)$,

257

258

259

260

261

262

263

264

265

266

267 268 269 270 27

272 (Gutiérrez et al., 2013; Ma et al., 2014). Because this gene is used for phylogeny of a variety of phages, 
273 we attempted to construct Propionibacterium phage phylogeny in a similar manner (Ganz et al., 2014; Li 274 et al., 2014), but were ultimately unsuccessful due to the lack of a full-length de novo assembled reference 275 genes in the dataset.

276

277 Most skin HPVs were identified as gamma HPVs, the prototypical cutaneous HPV class (Figure 1B) 278 (Mistry, Wibom \& Evander, 2008). Few contigs were identified as beta and $\mathrm{Mu} / \mathrm{Nu} \mathrm{HPVs}$, and none were 279 identified as alpha HPVs. This is consistent with data from the Human Microbiome Project cohort (Ma et 280 al., 2014).

281

282 Fewer Staphylococcus phage marker genes were identified, compared to HPVs, likely because 283 Staphylococcus phage genomes are orders of magnitude longer than HPV genomes, thereby decreasing 284 the probability that contigs covered the entire genome. Because multiple displacement amplification 285 (MDA) was not used to create this dataset, there is no MDA-associated bias toward small circular 286 genomes. The Staphylococcus phage contigs belonged to the Phietalikevirus genus and orphan virus groups (those that have not yet been classified) (Figure 1C). Of the Staphylococcus phage contigs identified, $49.6 \%$ (123 out of 248 contigs) were predicted to be lysogenic, based on similarity to lysogenic phages in the ACLAME database, integrase genes in the Uniprot database, and Staphylococcus reference genomes from the NCBI nucleotide database, as described previously (Leplae, Lima-Mendez \& Toussaint, 2010; Minot et al., 2011; Hannigan et al., 2015). This is a minimum estimate of contig 292 lysogeny, as some of the other contigs may have lysogenic signatures that we failed to identify. 293 Furthermore, because this classification strategy is based on blast assignments, it may result in false 294 positives if genes in the database are homologous to genes present in lytic phages.

\section{Hypervariable Loci Within the Skin Virome}

297 We implemented a geometric distribution-based approach to identify regions of high genomic diversity, 298 as in (Zheng et al., 2010). Regions within each contig that contained a significantly higher frequency of 
299 SNPs over the stochastic background were identified as viral hypervariable loci. Significance was defined 300 as the frequency of SNPs having less than a 5\% chance of randomly occurring, given the geometric 301 distribution of the dataset. HPVs and Staphylococcus phages maintained 106 and 465 hypervariable loci, 302 respectively. We were unable to detect hypervariable loci in the Propionibacterium phage population.

303

304 To determine the virus protein family domains hosting hypervariable loci, we used the Hidden Markov 305 Model (HMM) analysis implemented by HMMer (Finn, Clements \& Eddy, 2011). Hypervariable loci306 containing HPV genes include E6, E2, and E1 genes, which are associated with infectious gene 307 expression, and the L1 major capsid gene, which is involved in tropism and host immune evasion (Table 308 S1). The L1 major capsid protein is also a target in contemporary, widely used HPV vaccines (Schiller \& 309 Lowy, 2012). Hypervariable loci were detected in a variety of Staphylococcus phage genes with predicted functions related to tropism, host immune evasion, and utilization of host resources (Table S2).

\section{Selective Pressures on Hypervariable Loci}

313 We evaluated the selective pressures on virus genes by calculating the $p \boldsymbol{N} / \boldsymbol{p} S$ ratio of non-synonymous 314 SNPs $(p \boldsymbol{N})$ to synonymous SNPs $(\boldsymbol{p S})$ within each virus taxa (Schloissnig et al., 2013). This was used as 315 an alternative to $\boldsymbol{d} \boldsymbol{N} / \boldsymbol{d} \boldsymbol{S}$ because $\boldsymbol{d} \boldsymbol{N} / \boldsymbol{d} \boldsymbol{S}$ assumes haplotype information which cannot be fulfilled by 316 metagenomic data (Schloissnig et al., 2013). In the $\boldsymbol{p N} / \boldsymbol{p} \boldsymbol{S}$ calculation, neutral evolution is defined as an 317 equal frequency of synonymous and non-synonymous polymorphisms. Selective pressure favors non318 synonymous mutations, resulting in increased $p N / p S$ ratios. Purifying selection has the opposite effect. 319 Because the existing model (Schloissnig et al., 2013) is susceptible to stochastic effects and extreme outliers (e.g. division by zero when $\boldsymbol{p S}=0$ ), we added a pseudocount correction (Formula 1).

322 We determined whether hypervariable loci are in fact loci of focused selective pressure by comparing $323 p N / p S$ values of the loci to the adjacent genomic regions. $p N / p S$ values of hypervariable loci were 324 significantly lower than adjacent regions in both HPV (median: adjacent $=1.0$, hypervariable loci $=0.21$; 
325 p-value $=3.4 \mathrm{e}-17$ ) and Staphylococcus phage (median: adjacent $=0.76$, hypervariable loci $=0.41 ; \mathrm{p}$-value $326=1.8 \mathrm{e}-40)$ genomes, suggesting purifying selection and a propensity to maintain existing protein 327 sequences (Figure 1D-E). HPV hypervariable loci were under significantly greater purifying selection 328 than those of Staphylococcus phages (median: HPV $=0.21$, Staphylococcus Phage $=0.41$; $\mathrm{p}$-value $=$ 329 4.64e-9) (Figure S1). Furthermore, not only are the $\mathrm{pN} / \mathrm{pS}$ values of the hypervariable significantly lower 330 than their adjacent regions, but very few of the loci have a $\mathrm{pN} / \mathrm{pS}$ value greater than one.

332 To evaluate whether the observed selective pressure in HPV and Staphylococcus virus communities is 333 genome-wide or localized to hypervariable loci, we quantified the selective pressure on each virus' 334 genome by calculating the overall $p N / p S$ ratio including hypervariable loci and non-hypervariable loci SNPs. We observed nearly neutral pressure across HPVs and Staphylococcus phages that mirrored pressures to those observed in the regions adjacent to the hypervariable loci (median: HPV $=1.0$, Staphylococcus phage $=0.81$, p-value $=3.2 \mathrm{e}-5)($ Figure $\mathbf{S 2})$.

\section{Functional Implications of Targeted Substitutions Within Hypervariable Loci}

In order to evaluate the specific nucleotide changes occurring at hypervariable loci, as well as to evaluate

341 the implications of specific nucleotide polymorphisms, we quantified the frequency of individual nucleotide substitutions within hypervariable loci. $\mathrm{A}>\mathrm{C}$ and $\mathrm{T}>\mathrm{C}$ substitutions were most frequent in HPV hypervariable loci (Figure 2A). Staphylococcus phages exhibited a significantly different substitution profile $(\mathrm{p}$-value $=0.00018$, Chi-Square test), with the most common substitutions being $\mathrm{A}>\mathrm{G}$ and G $>$ A transitions (Figure 2B). HPV and Staphylococcus phage substitutions were more likely to be transitions, with a transition/transversion (ti/tv) ratio of 3.25 and 2.02 , respectively.

348 We predicted how hypervariable loci SNPs might affect protein functionality by evaluating patterns of the 349 amino acid substitutions while correcting for the random chance that the substitution will occur. The most 350 frequent non-synonymous amino acid substitution in HPVs was glycine (consensus amino acid) to valine 
351 (variant amino acid, Figure 2C). While these amino acids are (nonpolar and hydrophobic), glycine is less

352 hydrophobic than valine. The most frequent non-synonymous amino acid substitution in Staphylococcus

353 phages was proline to leucine (Figure 2D), a substitution between a non-polar cyclic amino acid and an

354 aliphatic straight chain amino acid. Profiles of amino acid substitution were significantly different

355 between HPVs and Staphylococcus phages ( $\mathrm{p}$-value $=0.0021$; Chi-square test).

357 Amino acid polarity and charge were largely maintained in HPV hypervariable loci (Figure 2E, G). In

358 instances of altered charge, visual inspection suggests the most frequent changes were from neutral to 359 positive or negative charge, or positive to neutral charge. Consensus acidic polar residues were not 360 associated with polymorphisms. Staphylococcus phage community hypervariable loci appeared to be

361

362

363

364

365

366

367

368

369

370

371

372

373

374 375 376

under weaker substitution selection, with a greater diversity in amino acid charge and polarity (Figure

2F, H) compared to HPV. Patterns of substitution charge and polarity were not significant (p-value $>0.5$; Chi-square test) when comparing the entire HPV to Staphylococcus phage substitution profiles.

We reinforced the observed functional implications of hypervariable loci by predicting the effects of their associated single amino acid variants (SAVs) on gene phenotype using the support vector machine algorithm implemented in SuSPect (Yates et al., 2014). This method assigns a deleterious score to each hypervariable loci SNP-associated SAV, with 0 representing a neutral SAV and 100 representing a SAV with high likelihood to impact phenotype. These scores are based on the predicted impact of the single amino acid variant on the tertiary and secondary structure of the resulting protein, the location of the SAV within the resulting protein (surface vs core), and whether the SAV has previously been associated with altered protein-protein interactions. Both Staphylococcus phages and HPVs have an abundance of SNPs associated with SAVs predicted to be deleterious (deleterious scores approaching 100) (Figure 3). The HPV SNPs were predicted to be significantly more likely to impact phenotype than the Staphylococcus phage SNPs (median: HPV $=45$, Staphylococcus phage $=17$; p-value $<2.2 \mathrm{e}-16$ ), suggesting that SNPs impact functionality differently between viruses.

Peer] reviewing PDF | (2016:09:13597:2:0:NEW 3 Jan 2017) 
378 Diversity Generating Retroelements as a Mechanism for Targeted Hypervariability

379 Diversity generating retroelements (DGRs) are a genetic system used by bacteriophages (as well as 380 bacteria and archea) to promote targeted hypervariability in genes (Doulatov et al., 2004). While DGRs 381 are complex and consist of many components, at their most basic they can be identified as elements 382 consisting of a reverse transcriptase gene and a repeated nucleotide sequence of length $<150 \mathrm{bp}$ that is 383 found in two separate locations of the genome (Doulatov et al., 2004; Minot et al., 2012; Schillinger et al., 384 2012), termed the template region and the variable region. The template region is transcribed, then reverse 385 transcribed in an error-prone fashion. The resulting cDNA is then integrated into the variable region, 386 introducing base substitutions. Targeted hypervariation impacts functions including broadened host cell tropism by mutagenizing a phage tail fiber gene (Doulatov et al., 2004).

We thus sought to identify candidate DGR cassettes within our viral contigs. We defined the candidate transcriptase gene. We only considered cassettes that were located within a predicted viral gene, contained at least one hypervariable locus in their variable region, and exhibited truly random variation (different between reads). Based on these criteria, we identified one Staphylococcus phage DGR candidate that contained hypervariable loci. We also identified five other DGR candidates that were associated with hypervariable loci outside of predicted genes or that failed to demonstrate linkage disequilibrium, suggesting an association with cryptic genes or pseudogenes.

399 For the Staphylococcus phage DGR candidate with hypervariable loci, we calculated the linkage 400 disequilibrium associated with the variable nucleotide positions to infer whether the DGR was active or 401 inactive (e.g. an evolutionary artifact). The DGR cassette had unlinked nucleotide variation, which was 402 supported by low levels of linkage disequilibrium (squared allelic correlation $\mathrm{R}^{2}$ ) between SNP pairs in 
403 the variable region (Figure 4). In this cassette, the template region has less frequent blocks of linkage 404 equilibrium (unlinked variants) while the variable region was associated with greater linkage equilibrium. 405 Together this suggests the observed Staphylococcus phage is active. The variable region was associated 406 with a gene of unknown function.

407

408 Skin Virome Variability Patterns and SNP Locations Are Reproducible Across Different Datasets

409 We repeated our analyses in a separate, independently collected dataset from another research group 410 (SRA BioProject: 46333) (Oh et al., 2014) to determine the generalizability of our findings. We analyzed 411 metagenomic sequence data of skin specimens that were collected from the retroauricular crease without 412 initial purification of virus like particles. Consistent with our primary analysis, Staphylococcus and 413 Propionibacterium phages were identified as having the highest coverage and similarity to reference genomes (Figure 5A). Pseudomonas phages were identified but were in the minority and had low coverage and similarity to reference genomes. HPV was not identified as a major virus in our analysis of the retroauricular crease; however, molluscum contagiosum virus, a poxvirus that causes cutaneous growths that become severe in immunocmpromised states, was present in high relative abundance in agreement with the original published findings (Oh et al., 2014).

419

Similar to our primary analysis, we identified 158 hypervariable loci within the Staphylococcus phage communities, and observed only 12 hypervariable loci associated with Propionibacterium phages, further highlighting the overall lack of genetic variability of the Propionibacterium phage communities. The Staphylococcus phage hypervariable loci were associated with purifying selection, yielding a $\mathrm{pN} / \mathrm{pS}$ ratio slightly below 0.4 (Figure 5B), recapitulating our findings in the primary dataset (Figure 1E).

426 Staphylococcus phage nucleotide substitutions were associated with transitions between guanine and 427 adenine residues, as we observed in our primary analysis (Figure 5C). The ti/tv ratio of these loci was 428 1.02. The most common amino acid substitution was proline to leucine (Figure 5D) and the substitution 
429 properties appeared loosely specific based on charge and polarity (Figure 5E-F), reproducing our 430 findings (Figure 2).

431

432 We also evaluated the reproducibility of SNP position between identical but independently assembled 433 genomic contigs of the two studies. We quantified the proportion of SNPs in our primary dataset that 434 were also found at the same position in the secondary dataset. This revealed a median of approximately $43550 \%$ overlap between datasets (Figure 5G). As a control, we generated a simulated dataset using 436 randomly assigned SNP positions instead of those determined experimentally. This yielded a significantly 437 lower median of approximately 15\% shared nucleotide SNP calls (Figure 5G), suggesting that the 438 observed SNP position is not random. These data indicate that our findings are consistent across different 439 skin virome populations and techniques of collection and sequencing.

\section{DISCUSSION}

442 Here we report localized targeted hypervariability in some of the most prevalent members of the skin 443 virome. Hypervariable loci provide a substrate for complex virus evolution throughout the virome, which 444 manifest as natural selection that differs by virus type and enforces purifying selection. Hypervariable 445 loci, which were present in genes encoding factors including virus tropism and host immune evasion, and 446 were primarily under purifying selection, whereas overall virus genomes were under near neutral 447 selection. We characterized selected substitution of nucleotides within hypervariable loci, with different 448 variant patterns between HPVs and Staphylococcus bacteriophage communities. These findings were 449 validated in an independently collected cohort.

451 We showed Propionibacterium phages exhibited strikingly low nucleotide variation with nearly no 452 identifiable hypervariable loci. While this starkly contrasts with HPVs and Staphylococcus 453 bacteriophages, it agrees with our current understanding of Propionibacterium phage diversity. Genome 454 comparisons of Propionibacterium phage isolates revealed minimal nucleotide diversity, although this 
455 has yet to be supported by targeted metagenomic evidence such as presented here (Marinelli et al., 2012;

456 Liu et al., 2015). The lack of Propionibacterium phage hypervariability in our metagenomic dataset 457 provides another level of evidence for minimal Propionibacterium phage diversity on the skin.

459 There are several potential factors that could contribute to the limited diversity of Propionibacterium 460 phages, and a consensus has yet to be reached. The lack of hypervariable loci suggests minimal 461 evolutionary pressure on the phages, which may be a reflection of their environment. As suggested 462 previously, the phages and their hosts reside in a unique and relatively isolated environment deeper in the 463 skin, which may contribute to low genomic diversity (Marinelli et al., 2012). Our data further support this 464 hypothesis. Another factor that could contribute to differential phage genomic diversity is their host 465 range. Although Propionibacterium phages have broad infectious capabilities within bacterial species, 466 they may be limited in their ability to infect other species (Marinelli et al., 2012). Staphylococcus phages 467 demonstrate greater genomic diversity, and may be capable of infecting a broader range of hosts.

468

469 We observed greater selective pressure on HPVs compared to Staphylococcus phages, which may reflect 470 greater pressures from the human immune system, compared to phage bacterial hosts. This may also 471 reflect the effects of different virus replication-cycles on evolutionary selection. HPVs do not usually 472 exist in a latent, integrated state, while Staphylococcus phages do (Bae et al., 2006; Goerke et al., 2009; 473 Edwards et al., 2013). Our data suggest that at least one half of the observed Staphylococcus phages have 474 temperate replication-cycles. As long as the Staphylococcus phages are integrated into the bacterial 475 genome, we hypothesize that they are under less selective pressure by external factors.

476

477 The viral hypervariable loci are primarily associated with purifying selective pressure, a finding in 478 agreement with previous non-metagenomic virus reports (Chen et al., 2005; Wolf et al., 2006; Li et al., 479 2011). The observed prominent purifying selective pressure supports an evolutionary model of long static 480 periods punctuated by brief positive selection, as is observed in influenza virus (Wolf et al., 2006). Here 
481 nucleotide diversity acts as a primer for rapid virus adaptation through brief positive selection, while

482 maintaining periods of consistency through purifying selection during static environmental conditions. As

483 an example, some localized nucleotide diversity may allow for the generation of phages with different

484 tropisms (e.g. different bacterial strains). If there are limited hosts, the phages that successfully infect

485 those hosts will be selected for, and altered tropisms will be actively selected against. If that host

486 population changes, then those viruses with the appropriate tropism will be selected for instead of being

487 selected against. Ultimately, longitudinal and strain specific studies will be required to further address this

488 hypothesis.

489

490 The amino acid substitutions associated with hypervariable loci were non-random and followed virus-

491 specific substitution patterns (Figure 2). HPV hypervariable loci were most associated with substitutions

492 from glycine to valine. This substitution has recently been associated with infectious functionality,

493 whereby the introduction of this mutation resulted in impaired infective ability of the virus (Bronnimann

494 et al., 2013). This impaired infectious activity was attributed to a reduced efficacy of genomic DNA

495 endosomal translocation within the host, which may have been the result of impaired trans-membrane

496 alpha-helical self-association of the L2 minor capsid protein. Given these findings, our results suggest

497 hypervariable loci are involved in promoting diversity in endosomal translocation motifs to some degree.

498 Hypervariable loci may certainly have other diverse, functional roles, as evidenced by the wide range of

499 hypervariable loci-containing genes.

500

501 The dominant amino acid substitution observed in Staphylococcus bacteriophages was from proline to

502 leucine, a different substitution than that observed in HPV. This substitution could affect protein

503 structure, particularly a loss of rigidity due to the loss of the proline ring structure. This observation may

504 reflect a biologically important adaptation of the bacteriophage to its Staphylococcus host, which have

505 been shown to be auxotrophic for proline and leucine and may switch between auxotroph and prototroph

506 depending on nutrient availability (Emmett \& Kloos, 1975; Nuxoll et al., 2012). Because the amino acids 
507 may be in variable supply depending on the host, phages may alter their amino acid usage to exploit what

508 is most readily available.

509

510 The overall selective nucleotide substitutions associated with HPV amino acid charge highlights a

511 potential maintenance of HPV tropism. The lack of HPV substitutions between charges may suggest a

512 selection against strong alterations in protein isoelectric points, which have been implicated in affecting

513 HPV tropism (Mistry, Wibom \& Evander, 2008). Furthermore, because acidic residues almost never

514 mutated to non-polar residues, these acidic amino acids are potentially important external amino acids

515 that may participate in tropic protein-protein interactions.

516

517 The described patterns in our findings suggest a role for targeted and/or localized genomic variation. One

518

519

520

521

522

523

524

525

526

527

528

529

530

531 This study illustrates the diversity of evolutionary pressures on skin virus communities. It begins to

532 provide further community-wide context to the molecular understanding of skin viruses, and highlights 
533 important aspects of their infectious cycles. These insights also contribute to understanding virus ecology

534 of the human skin, and will inform future translational research into HPV vaccination, vaccination against

535 other skin-associated viruses, effects of phages on bacterial pathogenesis, and phage therapy.

536 Understanding how viruses evolve in their natural communities is crucial for improving these

537 translational applications, and our findings here, which focus on HPV and Staphylococcus phages, will

538 benefit cutaneous clinical virology and provide a foundation for future studies.

539

540 CONCLUSIONS

541 We report that the skin virus communities contain hypervariable loci that are associated with strong

542 purifying selection and targeted nucleotide substitution. The degree of selective pressure and impact of

543 amino acid substitutions on protein chemistry (structure, isoelectric point, polarity) is virus specific,

544 despite being members of the same community. These hypervariable loci are found within diverse viral

545 strains, with varying degrees of phylogenetic divergence over their evolutionary history. We further

546 reproduce these findings in independently collected skin virus communities.

547

548 ACKNOWLEDGEMENTS

549 We thank the members of the Grice and Bushman laboratories for their underlying contributions. 


$$
\frac{p N}{p S}=\frac{\left(\frac{M_{N}+\frac{\sqrt[2]{\tilde{C}_{M}}}{2}}{\sum_{i=1}^{L} \frac{N_{i}}{3}}\right)}{\left(\frac{M_{S}+\frac{\sqrt[2]{\tilde{C}_{M}}}{2}}{\sum_{i=1}^{L} \frac{S_{i}}{3}}\right)}
$$

\section{Formula 1}

554 The formula used to calculate the $p N / p S$ ratio for a gene. $M_{N}$ is the number of non-synonymous SNPs

555 within the gene and $M_{S}$ is the number of synonymous SNPs found within the gene. Each mutation value is

556 normalized for the likelihood that the result would have happened by chance, calculated as the sum of the 557 proportions of nucleotides that would have resulted in either a non-synonymous or synonymous mutation.

558 To calculate this proportion, the possible non-synonymous mutations $(\mathrm{N})$ and synonymous mutations (S)

559 at position $\mathrm{i}$ within the gene are expressed as a fraction of the three possible alternate nucleotides. SNP 560 counts were smoothed as pseudo-counts using the median SNP sequence coverage $\left(C_{M}\right)$. 


\section{REFERENCES}

564 Bacher JM, Bull JJ, Ellington AD 2003. Evolution of phage with chemically ambiguous proteomes. $B M C$

565

566

567

568

569

570

571

572

573

574

575

576

577

578

579

580

581

582

583

584

585

586

587

588

589

590

591

592

593

594

595

596

597

598

599

600

601

602

603

604

605

606

607

608

609

610

611

612 Evolutionary Biology 3:24. DOI: 10.1186/1471-2148-3-24.

Bae T, Baba T, Hiramatsu K, Schneewind O 2006. Prophages of Staphylococcus aureus Newman and their contribution to virulence. Molecular Microbiology 62:1035-1047. DOI: 10.1111/j.13652958.2006.05441.x.

Bajgain P, Richardson BA, Price JC, Cronn RC, Udall JA 2011. Transcriptome characterization and polymorphism detection between subspecies of big sagebrush (Artemisia tridentata). BMC Genomics 12:370. DOI: 10.1186/1471-2164-12-370.

Boisvert S, Raymond F, Godzaridis E, Laviolette F, Corbeil J 2012. Ray Meta: scalable de novo metagenome assembly and profiling. Genome biology 13:R122. DOI: 10.1186/gb-2012-13-12-r122.

Borghans JAM, Beltman JB, De Boer RJ 2004. MHC polymorphism under host-pathogen coevolution. Immunogenetics 55:732-739. DOI: 10.1007/s00251-003-0630-5.

Bronnimann MP, Chapman JA, Park CK, Campos SK 2013. A transmembrane domain and GxxxG motifs within L2 are essential for papillomavirus infection. Journal of Virology 87:464-473. DOI: 10.1128/JVI.01539-12.

Camacho C, Coulouris G, Avagyan V, Ma N, Papadopoulos J, Bealer K, Madden TL 2009. BLAST+: architecture and applications. BMC Bioinformatics 10:1. DOI: 10.1186/1471-2105-10-421.

Chen Z, Terai M, Fu L, Herrero R, DeSalle R, Burk RD 2005. Diversifying selection in human papillomavirus type 16 lineages based on complete genome analyses. Journal of Virology 79:70147023. DOI: 10.1128/JVI.79.11.7014-7023.2005.

Das SR, Hensley SE, Ince WL, Brooke CB, Subba A, Delboy MG, Russ G, Gibbs JS, Bennink JR, Yewdell JW 2013. Defining influenza A virus hemagglutinin antigenic drift by sequential monoclonal antibody selection. Cell Host and Microbe 13:314-323. DOI: 10.1016/j.chom.2013.02.008.

de Villiers E-M, Fauquet C, Broker TR, Bernard H-U, Hausen zur H 2004. Classification of papillomaviruses. Virology 324:17-27. DOI: 10.1016/j.virol.2004.03.033.

Delcher AL, Bratke KA, Powers EC, Salzberg SL 2007. Identifying bacterial genes and endosymbiont DNA with Glimmer. Bioinformatics 23:673-679. DOI: 10.1093/bioinformatics/btm009.

Donaldson EF, Lindesmith LC, Lobue AD, Baric RS 2010. Viral shape-shifting: norovirus evasion of the human immune system. Nature Reviews Microbiology 8:231-241. DOI: 10.1038/nrmicro2296.

Doulatov S, Hodes A, Dai L, Mandhana N, Liu M, Deora R, Simons RW, Zimmerly S, Miller JF 2004. Tropism switching in Bordetella bacteriophage defines a family of diversity-generating retroelements. Nature 431:476-481. DOI: 10.1038/nature02833.

Edwards TG, Helmus MJ, Koeller K, Bashkin JK, Fisher C 2013. Human papillomavirus episome stability is reduced by aphidicolin and controlled by DNA damage response pathways. Journal of Virology 87:3979-3989. DOI: 10.1128/JVI.03473-12.

Emmett M, Kloos WE 1975. Amino acid requirements of staphylococci isolated from human skin. Canadian journal of microbiology 21:729-733. DOI: 10.1139/m75-107.

Finn RD, Clements J, Eddy SR 2011. HMMER web server: interactive sequence similarity searching. Nucleic Acids Research 39:W29-37. DOI: 10.1093/nar/gkr367.

Ganz HH, Law C, Schmuki M, Eichenseher F, Calendar R, Loessner MJ, Getz WM, Korlach J, Beyer W, Klumpp J 2014. Novel giant siphovirus from Bacillus anthracis features unusual genome characteristics. PLOS ONE 9:e85972. DOI: 10.1371/journal.pone.0085972.

Goerke C, Pantucek R, Holtfreter S, Schulte B, Zink M, Grumann D, Broker BM, Doskar J, Wolz C 2009. Diversity of Prophages in Dominant Staphylococcus aureus Clonal Lineages. Journal of Bacteriology 191:3462-3468. DOI: 10.1128/JB.01804-08.

Guan M, Wang W, Liu X, Tong Y, Liu Y, Ren H, Zhu S, Dubuisson J, Baumert TF, Zhu Y, Peng H, Aurelian L, Zhao P, Qi Z 2012. Three different functional microdomains in the hepatitis C virus hypervariable region 1 (HVR1) mediate entry and immune evasion. The Journal of biological 
613

614

615

616

617

618

619

620

621

622

623

624

625

626

627

628

629

630

631

632

633

634

635

636

637

638

639

640

641

642

643

644

645

646

647

648

649

650

651

652

653

654

655

656

657

658

659

660

661

662

663

chemistry 287:35631-35645. DOI: 10.1074/jbc.M112.382341.

Guo H, Arambula D, Ghosh P, Miller JF 2014. Diversity-generating Retroelements in Phage and Bacterial Genomes. Microbiology spectrum 2:1237-1252. DOI: 10.1128/microbiolspec.MDNA30029-2014.

Gutiérrez D, Adriaenssens EM, Martínez B, Rodríguez A, Lavigne R, Kropinski AM, García P 2013. Three proposed new bacteriophage genera of staphylococcal phages: "3alikevirus," "77likevirus" and "Phietalikevirus." Archives of Virology 159:389-398. DOI: 10.1007/s00705-013-1833-1.

Hannigan GD, Grice EA 2013. Microbial Ecology of the Skin in the Era of Metagenomics and Molecular Microbiology. Cold Spring Harbor Perspectives in Medicine 3:a015362-a015362. DOI: 10.1101/cshperspect.a015362.

Hannigan GD, Hodkinson BP, McGinnis K, Tyldsley AS, Anari JB, Horan AD, Grice EA, Mehta S 2014. Culture-independent pilot study of microbiota colonizing open fractures and association with severity, mechanism, location, and complication from presentation to early outpatient follow-up. Journal of Orthopaedic Research 32:597-605. DOI: 10.1002/jor.22578.

Hannigan GD, Meisel JS, Tyldsley AS, Zheng Q, Hodkinson BP, SanMiguel AJ, Minot S, Bushman FD, Grice EA 2015. The Human Skin Double-Stranded DNA Virome: Topographical and Temporal Diversity, Genetic Enrichment, and Dynamic Associations with the Host Microbiome. mBio 6:e01578-15. DOI: 10.1128/mBio.01578-15.

Human Microbiome Project Consortium 2012. Structure, function and diversity of the healthy human microbiome. Nature 486:207-214. DOI: 10.1038/nature11234.

Katoh K, Standley DM 2013. MAFFT multiple sequence alignment software version 7: improvements in performance and usability. Molecular Biology and Evolution 30:772-780. DOI: 10.1093/molbev/mst010.

Kearse M, Moir R, Wilson A, Stones-Havas S, Cheung M, Sturrock S, Buxton S, Cooper A, Markowitz S, Duran C, Thierer T, Ashton B, Meintjes P, Drummond A 2012. Geneious Basic: an integrated and extendable desktop software platform for the organization and analysis of sequence data. Bioinformatics 28:1647-1649. DOI: 10.1093/bioinformatics/bts199.

Koboldt DC, Zhang Q, Larson DE, Shen D, McLellan MD, Lin L, Miller CA, Mardis ER, Ding L, Wilson RK 2012. VarScan 2: somatic mutation and copy number alteration discovery in cancer by exome sequencing. Genome Research 22:568-576. DOI: 10.1101/gr.129684.111.

Kubinak JL, Ruff JS, Hyzer CW, Slev PR, Potts WK 2012. Experimental viral evolution to specific host MHC genotypes reveals fitness and virulence trade-offs in alternative MHC types. Proceedings of the National Academy of Sciences of the United States of America 109:3422-3427. DOI: 10.1073/pnas.1112633109.

Langille MGI, Zaneveld J, Caporaso JG, McDonald D, Knights D, Reyes JA, Clemente JC, Burkepile DE, Thurber RLV, Knight R, Beiko RG, Huttenhower C 2013. Predictive functional profiling of microbial communities using 16S rRNA marker gene sequences. Nature Biotechnology:1-10. DOI: 10.1038/nbt.2676.

Langmead B, Salzberg SL 2012. Fast gapped-read alignment with Bowtie 2. Nature Methods 9:357-359. DOI: $10.1038 /$ nmeth.1923.

Leplae R, Lima-Mendez G, Toussaint A 2010. ACLAME: a CLAssification of Mobile genetic Elements, update 2010. Nucleic Acids Research 38:D57-61. DOI: 10.1093/nar/gkp938.

Li H, Handsaker B, Wysoker A, Fennell T, Ruan J, Homer N, Marth G, Abecasis G, Durbin R, 1000 Genome Project Data Processing Subgroup 2009. The Sequence Alignment/Map format and SAMtools. Bioinformatics 25:2078-2079. DOI: 10.1093/bioinformatics/btp352.

Li S, Fan H, An X, Fan H, Jiang H, Chen Y, Tong Y 2014. Scrutinizing virus genome termini by highthroughput sequencing. PLOS ONE 9:e85806. DOI: 10.1371/journal.pone.0085806.

Li W, Shi W, Qiao H, Ho SYW, Luo A, Zhang Y, Zhu C 2011. Positive selection on hemagglutinin and neuraminidase genes of H1N1 influenza viruses. Virology Journal 8:183. DOI: 10.1186/1743-422X8-183.

Lim ES, Zhou Y, Zhao G, Bauer IK, Droit L, Ndao IM, Warner BB, Tarr PI, Wang D, Holtz LR 2015.

Peer] reviewing PDF | (2016:09:13597:2:0:NEW 3 Jan 2017) 
664

665

666

667

668

669

670

671

672

673

674

675

676

677

678

679

680

681

682

683

684

685

686

687

688

689

690

691

692

693

694

695

696

697

698

699

700

701

702

703

704

705

706

707

708

709

710

711

712

713

714

Early life dynamics of the human gut virome and bacterial microbiome in infants. Nature Medicine. DOI: $10.1038 / \mathrm{nm} .3950$.

Liu J, Yan R, Zhong Q, Ngo S, Bangayan NJ, Nguyen L, Lui T, Liu M, Erfe MC, Craft N, Tomida S, Li $\mathrm{H}$ 2015. The diversity and host interactions of Propionibacterium acnes bacteriophages on human skin. The ISME Journal:1-16. DOI: 10.1038/ismej.2015.47.

Ly M, Abeles SR, Boehm TK, Robles-Sikisaka R, Naidu M, Santiago-Rodriguez T, Pride DT 2014. Altered Oral Viral Ecology in Association with Periodontal Disease. mBio 5:e01133-14-e01133-14. DOI: $10.1128 / \mathrm{mBio} .01133-14$.

Ma Y, Madupu R, Karaoz U, Nossa CW, Yang L, Yooseph S, Yachimski PS, Brodie EL, Nelson KE, Pei Z 2014. Human Papillomavirus Community in Healthy Persons, Defined by Metagenomics Analysis of Human Microbiome Project Shotgun Sequencing Data Sets. Journal of Virology 88:4786-4797. DOI: 10.1128/JVI.00093-14.

Malim MH, Emerman M 2001. HIV-1 sequence variation: drift, shift, and attenuation. Cell 104:469-472.

Marinelli LJ, Fitz-Gibbon S, Hayes C, Bowman C, Inkeles M, Loncaric A, Russell DA, Jacobs-Sera D, Cokus S, Pellegrini M, Kim J, Miller JF, Hatfull GF, Modlin RL 2012. Propionibacterium acnes Bacteriophages Display Limited Genetic Diversity and Broad Killing Activity against Bacterial Skin Isolates. mBio 3:e0279-12-e00279-12. DOI: 10.1128/mBio.00279-12.

Meisel JS, Hannigan GD, Tyldsley AS, SanMiguel AJ, Hodkinson BP, Zheng Q, Grice EA 2016. Skin microbiome surveys are strongly influenced by experimental design. Journal of Investigative Dermatology. DOI: 10.1016/j.jid.2016.01.016.

Minot S, Bryson A, Chehoud C, Wu GD, Lewis JD, Bushman FD 2013. Rapid evolution of the human gut virome. Proceedings of the National Academy of Sciences of the United States of America 110:12450-12455. DOI: 10.1073/pnas.1300833110.

Minot S, Grunberg S, Wu GD, Lewis JD, Bushman FD 2012. Hypervariable loci in the human gut virome. Proceedings of the National Academy of Sciences of the United States of America 109:39623966. DOI: 10.1073/pnas.1119061109.

Minot S, Sinha R, Chen J, Li H, Keilbaugh SA, Wu GD, Lewis JD, Bushman FD 2011. The human gut virome: Inter-individual variation and dynamic response to diet. Genome Research 21:1616-1625. DOI: $10.1101 /$ gr.122705.111.

Mistry N, Wibom C, Evander M 2008. Cutaneous and mucosal human papillomaviruses differ in net surface charge, potential impact on tropism. Virology Journal 5:118. DOI: 10.1186/1743-422X-5118.

Nishida K, Frith MC, Nakai K 2009. Pseudocounts for transcription factor binding sites. Nucleic Acids Research 37:939-944. DOI: 10.1093/nar/gkn1019.

Norman JM, Handley SA, Baldridge MT, Droit L, Liu CY, Keller BC, Kambal A, Monaco CL, Zhao G, Fleshner P, Stappenbeck TS, McGovern DPB, Keshavarzian A, Mutlu EA, Sauk J, Gevers D, Xavier RJ, Wang D, Parkes M, Virgin HW 2015. Disease-specific alterations in the enteric virome in inflammatory bowel disease. Cell 160:447-460. DOI: 10.1016/j.cell.2015.01.002.

Novaes E, Drost DR, Farmerie WG, Pappas GJ, Grattapaglia D, Sederoff RR, Kirst M 2008. Highthroughput gene and SNP discovery in Eucalyptus grandis, an uncharacterized genome. $B M C$ Genomics 9:312. DOI: 10.1186/1471-2164-9-312.

Nuxoll AS, Halouska SM, Sadykov MR, Hanke ML, Bayles KW, Kielian T, Powers R, Fey PD 2012. CcpA regulates arginine biosynthesis in Staphylococcus aureus through repression of proline catabolism. PLoS Pathogens 8:e1003033. DOI: 10.1371/journal.ppat.1003033.

Oh J, Byrd AL, Deming C, Conlan S, Program NCS, Kong HH, Segre JA 2014. Biogeography and individuality shape function in the human skin metagenome. Nature 514:59-64. DOI: 10.1038/nature13786.

Quint KD, Genders RE, de Koning MN, Borgogna C, Gariglio M, Bouwes Bavinck JN, Doorbar J, Feltkamp MC 2015. Human Beta-papillomavirus infection and keratinocyte carcinomas. The Journal of Pathology 235:342-354. DOI: 10.1002/path.4425.

Rambaut A FigTree. 
715

716

717

718

719

720

721

722

723

724

725

726

727

728

729

730

731

732

733

734

735

736

737

738

739

740

741

742

743

744

745

746

747

748

749

750

751

752

753

754

755

756

757

758

759

Schiller JT, Lowy DR 2012. Understanding and learning from the success of prophylactic human papillomavirus vaccines. Nature Reviews Microbiology 10:681-692. DOI: 10.1038/nrmicro2872.

Schillinger T, Lisfi M, Chi J, Cullum J, Zingler N 2012. Analysis of a comprehensive dataset of diversity generating retroelements generated by the program DiGReF. BMC Genomics 13:1-1. DOI: 10.1186/1471-2164-13-430.

Schloissnig S, Arumugam M, Sunagawa S, Mitreva M, Tap J, Zhu A, Waller A, Mende DR, Kultima JR, Martin J, Kota K, Sunyaev SR, Weinstock GM, Bork P 2013. Genomic variation landscape of the human gut microbiome. Nature 493:45-50. DOI: 10.1038/nature11711.

Schloss PD, Handelsman J 2008. A statistical toolbox for metagenomics: assessing functional diversity in microbial communities. BMC Bioinformatics 9:34-15. DOI: 10.1186/1471-2105-9-34.

Schmieder R, Edwards R 2011. Fast identification and removal of sequence contamination from genomic and metagenomic datasets. PLOS ONE 6:e17288. DOI: 10.1371/journal.pone.0017288.

Shah S, Alexaki A, Pirrone V, Dahiya S, Nonnemacher MR, Wigdahl B 2014. Functional properties of the HIV-1 long terminal repeat containing single-nucleotide polymorphisms in Sp site III and CCAAT/enhancer binding protein site I. Virology Journal 11:92. DOI: 10.1186/1743-422X-11-92.

Shin JH, Blay S, McNeney B, Graham J 2006. LDheatmap: an R function for graphical display of pairwise linkage disequilibria between single nucleotide polymorphisms. Journal of Statistical ....

Stamatakis A 2014. RAxML version 8: a tool for phylogenetic analysis and post-analysis of large phylogenies. Bioinformatics 30:1312-1313. DOI: 10.1093/bioinformatics/btu033.

UniProt Consortium 2014. Activities at the Universal Protein Resource (UniProt). Nucleic Acids Research 42:D191-8. DOI: 10.1093/nar/gkt1140.

Van Doorslaer K, Tan Q, Xirasagar S, Bandaru S, Gopalan V, Mohamoud Y, Huyen Y, McBride AA 2013. The Papillomavirus Episteme: a central resource for papillomavirus sequence data and analysis. Nucleic Acids Research 41:D571-8. DOI: 10.1093/nar/gks984.

Varga M, Kuntová L, Pantůček R, Mašlaňová I, Růžičková V, Doškař J 2012. Efficient transfer of antibiotic resistance plasmids by transduction within methicillin-resistant Staphylococcus aureus USA300 clone. FEMS Microbiology Letters 332:146-152. DOI: 10.1111/j.1574-6968.2012.02589.x.

Vinzón SE, Braspenning-Wesch I, Müller M, Geissler EK, Nindl I, Gröne H-J, Schäfer K, Rösl F 2014. Protective vaccination against papillomavirus-induced skin tumors under immunocompetent and immunosuppressive conditions: a preclinical study using a natural outbred animal model. PLoS Pathogens 10:e1003924. DOI: 10.1371/journal.ppat.1003924.

Wang J, Aldabagh B, Yu J, Arron ST 2014. Role of human papillomavirus in cutaneous squamous cell carcinoma: a meta-analysis. Journal of the American Academy of Dermatology 70:621-629. DOI: 10.1016/j.jaad.2014.01.857.

Warnes G, Gorjanc G, Leisch F, Man M Genetics: Population Genetics.

Wolf YI, Viboud C, Holmes EC, Koonin EV, Lipman DJ 2006. Long intervals of stasis punctuated by bursts of positive selection in the seasonal evolution of influenza A virus. Biology direct 1:34. DOI: 10.1186/1745-6150-1-34.

Yates CM, Filippis I, Kelley LA, Sternberg MJE 2014. SuSPect: enhanced prediction of single amino acid variant (SAV) phenotype using network features. Journal of Molecular Biology 426:2692-2701. DOI: 10.1016/j.jmb.2014.04.026.

Zheng Q, Ryvkin P, Li F, Dragomir I, Valladares O, Yang J, Cao K, Wang L-S, Gregory BD 2010. Genome-wide double-stranded RNA sequencing reveals the functional significance of base-paired RNAs in Arabidopsis. PLoS Genetics 6:e1001141. DOI: 10.1371/journal.pgen.1001141. 


\section{Figure 1 (on next page)}

Phylogenetic \& Evolutionary Characteristics of Skin Virome Hypervariable Loci

A) Scatter plot depicting the candidate contigs considered for analysis in this study. Each point is a contig that mapped to a reference virus genome. The $x$-axis shows the length ( $\log 10$ scale) of the contig subsection that mapped to the reference genome. The $y$-axis shows the overall coverage of the contig, as a quantification of sequences aligning to the contig. The color highlights the reference virus genome that the contig was most similar to, and the size depicts the e-value (inverse $\log 10$ ) associated with the contig-reference match. The horizontal dashed line marks the threshold of 10X coverage, and the vertical dashed line marks the $750 \mathrm{bp}$ length threshold. B) Phylogenetic tree of skin virome HPVs and (C) Staphylococcus phages, structured onto a standard phylogenetic tree using reference genomes. HPV phylogeny was based on the L1 major capsid gene and Staphylococcus phage phylogeny was based on the large terminase subunit. Contigs from this study are highlighted as orange dots, and genera are labeled with text. Phylogenetic lengths were normalized to ranks to facilitate visualization. D) Box plots depicting the evolutionary pressure of HPVs (left) and Staphylococcus bacteriophages (right) at the hypervariable loci (blue) and the regions immediately adjacent to the hypervariable loci (red). Adjacent regions were calculated as being twice the length of the hypervariable loci (see visualization to the right). The hypervariable locus and adjacent region (combination of both sides) from each sample were evaluated for evolutionary pressure (y-axis) using SNPs (pink lines in right illustration). Asterisk $(*)$ indicates a statistically significant difference $(p<0.01)$. Notched boxplots were created using ggplot and show the median (center line), the inter-quartile range (IQR; upper and lower boxes), the highest and lowest value within $1.5 *$ IQR (whiskers), and the notch which provides an approximate $95 \%$ confidence interval as defined by $1.58 *$ IQR / sqrt(n). 


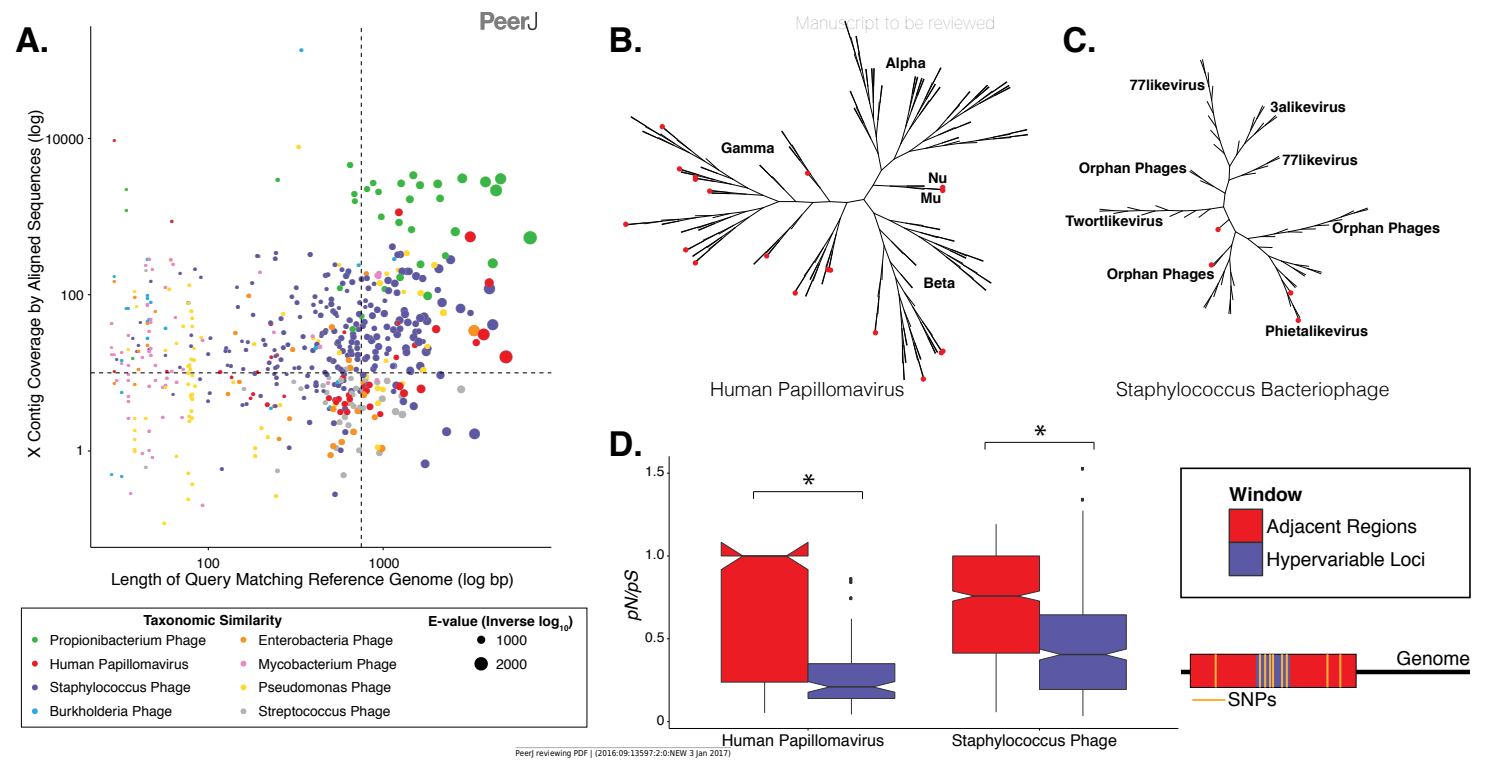




\section{Figure 2 (on next page)}

Nucleotide and Amino Acid Substitution Patterns within Viral Hypervariable Loci

Heat maps portraying the counts of every possible nucleotide substitution for each SNP found within (A) HPV and (B) Staphylococcus phage hypervariable loci. Tile color weight corresponds to the relative abundance of SNP substitution counts. The diagonal line highlights the panels associated with no substitution. The substitution patterns of amino acids at each SNP are also shown with exponential transformation (C,D). An illustration of the major amino acid substitutions are provided beneath the legends as a reference. Amino acid charge $(E, F)$ and polarity with acidity $(\mathrm{G}, \mathrm{H})$ are shown with $\log 10$ transformation. The absence of a basic or acidic polar identifier indicates the amino acid 20 is polar but neutral. The HPV substitution profiles are found in the left column and the Staphylococcus phage profiles are found on the right. Chi-Square significance $p$-value, comparing variation profiles between the viruses in each row (i.e. $A$ and $B$ ), is shown in the upper right corner of the associated Staphylococcus phage variation profile. The most frequently substituted amino acid pairs are highlighted with a box around the amino acid letters. 
Human Papillomavirus



E.

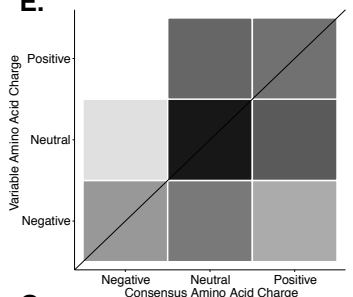

G



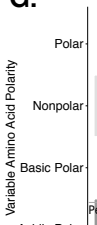

Acidic Polar
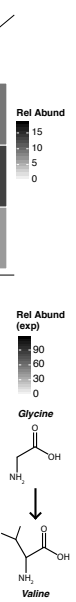

F
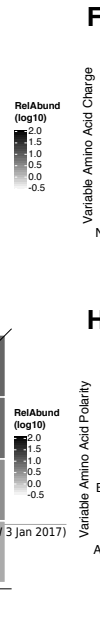

Staphylococcus Bacteriophage


D.
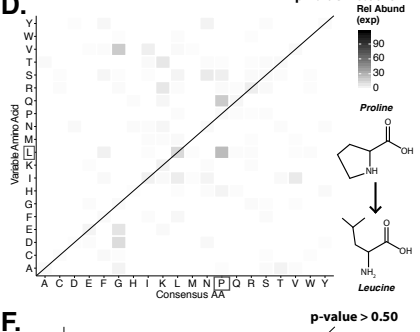

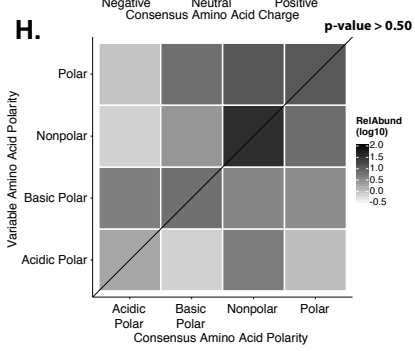


Figure 3 (on next page)

SVM Predicted Impact of Hypervariable Loci on Phenotype

Notched boxplot of deleterious scores in Human Papillomavirus (red) and Staphylococcus phage (blue) genomes. A low deleterious score indicates a predicted neutral phenotypic effect, while a high score indicates a predicted strong phenotypic effect. Asterisk $(*)$ indicates significant difference by Wilcoxon rank-sum test $(p<1 e 15)$. Boxplot parameters as described in figure 1. 
Impactful PeerJ

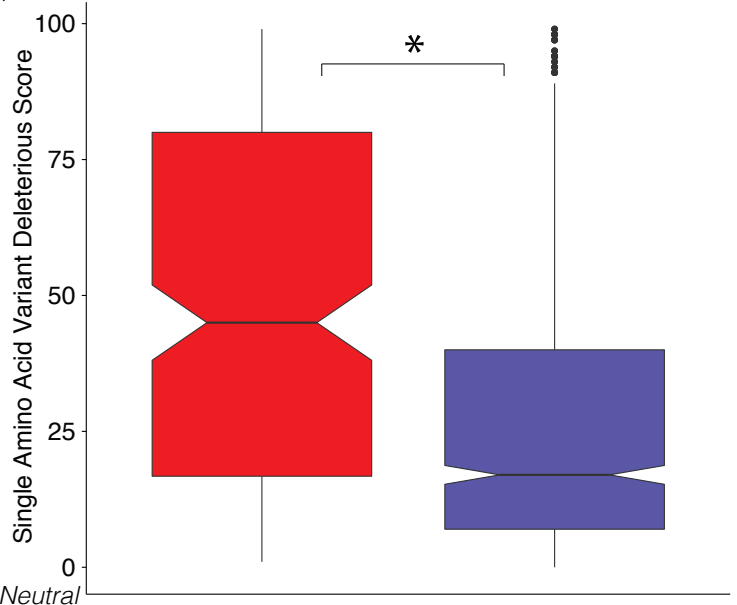

Human Papillomavirus Staphylococcus Phage 


\section{Figure 4 (on next page)}

The Diversity Generating Retroelement as a Mechanism for Targeted Nucleotide Variation

Alignment illustrating a putative diversity generating retroelement in Staphylococcus phage. Top Panel) Sashimi plot of sequence coverage across the contig. Coverage ranges from 0 $67 X$. Below the coverage is a map of the relevant genes predicted within the contig. Middle Panel) Sequence alignment of the diversity generating retroelement template region (left) and variable region (right). Bottom Panel) Linkage disequilibrium heatmap for the template and variable region. Panels compare variable nucleotides to each other and darker tiles indicate decreased linkage disequilibrium correlation, according to squared allelic correlation (R2) between pairs of SNPs . 
O

西 67
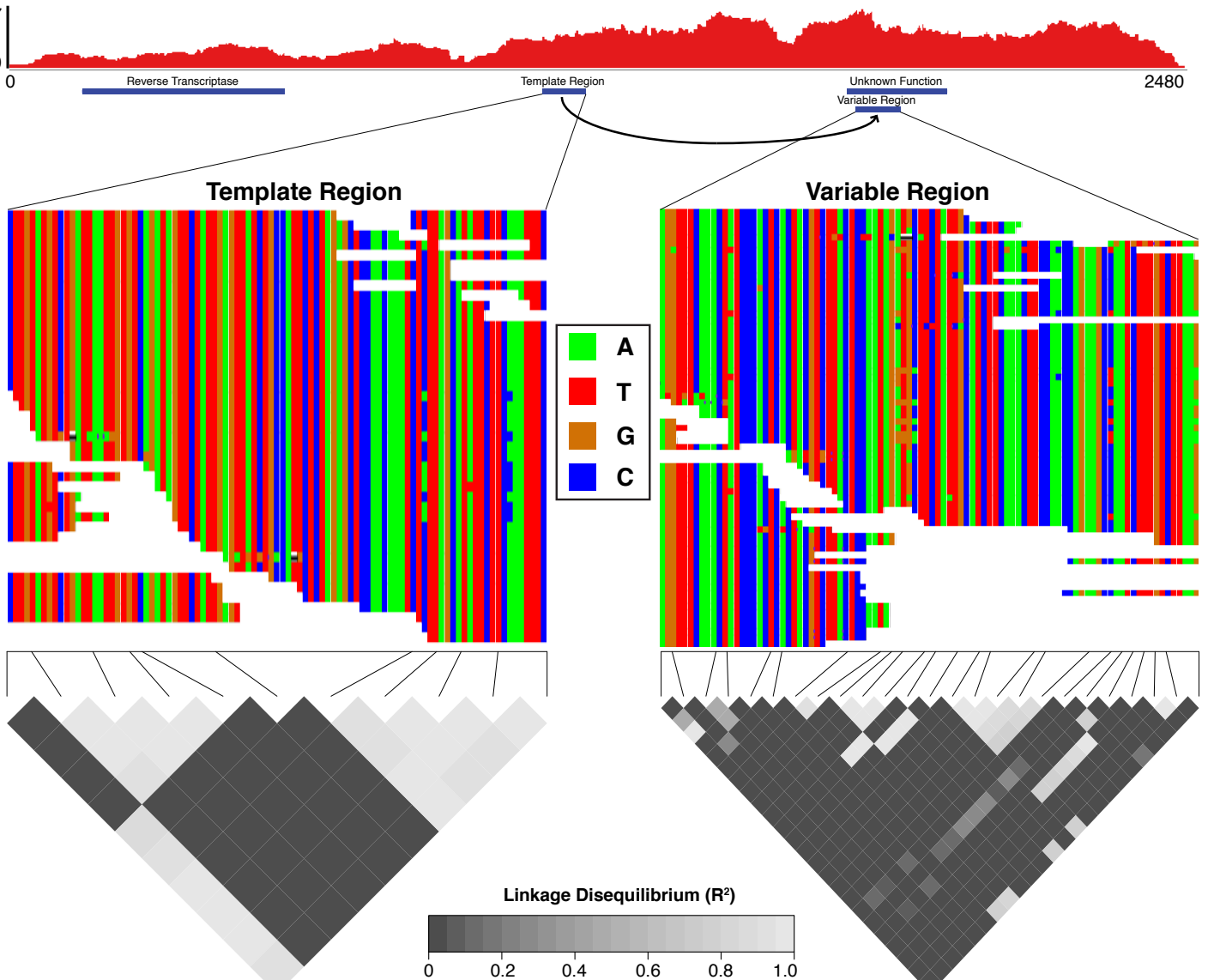

\section{Variable Region}

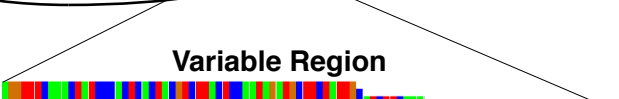

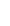

Linkage Disequilibrium $\left(\mathbf{R}^{2}\right)$

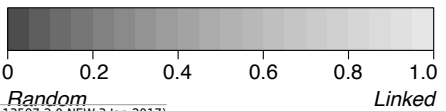




\section{Figure $\mathbf{5}$ (on next page)}

\section{Validation of Study Findings Using Secondary Dataset}

Results from the Oh et al dataset, which was analyzed using the same workflow as the primary dataset. A) Scatter plot depicting the candidate contigs considered for analysis in this study. Each point is a contig that mapped to a reference virus genome. The $x$-axis shows the length (in nucleotides) of the contig subsection that mapped to the reference genome. The $y$-axis shows the overall coverage of the contig as a quantification of sequences aligning to the contig. The color highlights the reference virus genome that the contig was most similar to, and the size depicts the blast bit score associated with the contig-reference match. B) Box plots depicting the evolutionary pressure of Staphylococcus bacteriophages at the hypervariable loci (blue) and the regions immediately adjacent to the hypervariable loci (red). C) Heat map portraying the counts of every possible nucleotide substitution for each SNP found within 21 Staphylococcus phage hypervariable loci. Tile color weight corresponds to the relative abundance of SNP substitution counts. The diagonal line highlights the panels associated with no substitution. The substitution patterns of amino acids at each (D) SNP, (E) amino acid charge, and (F) polarity with acidity are also shown. G) Notched boxplot illustrating the percent of primary dataset SNPs whose nucleotide positions were identical to those from the secondary validation sample set (left) compared to a simulated dataset of randomly assigned SNP locations (right). The inset shows an example contig identified in both datasets with $81 \%$ identical SNP positions. SNPs are represented as yellow lines, with the inner circle representing the validation dataset, and the middle circle representing the primary dataset. The outmost ring illustrates the contig, colored by nucleotides $(A=$ Red, $\mathrm{C}=\mathrm{Blue}, \mathrm{G}=$ Yellow, $\mathrm{T}=\mathrm{Green})$. Boxplot parameters as described in figure 1 . 

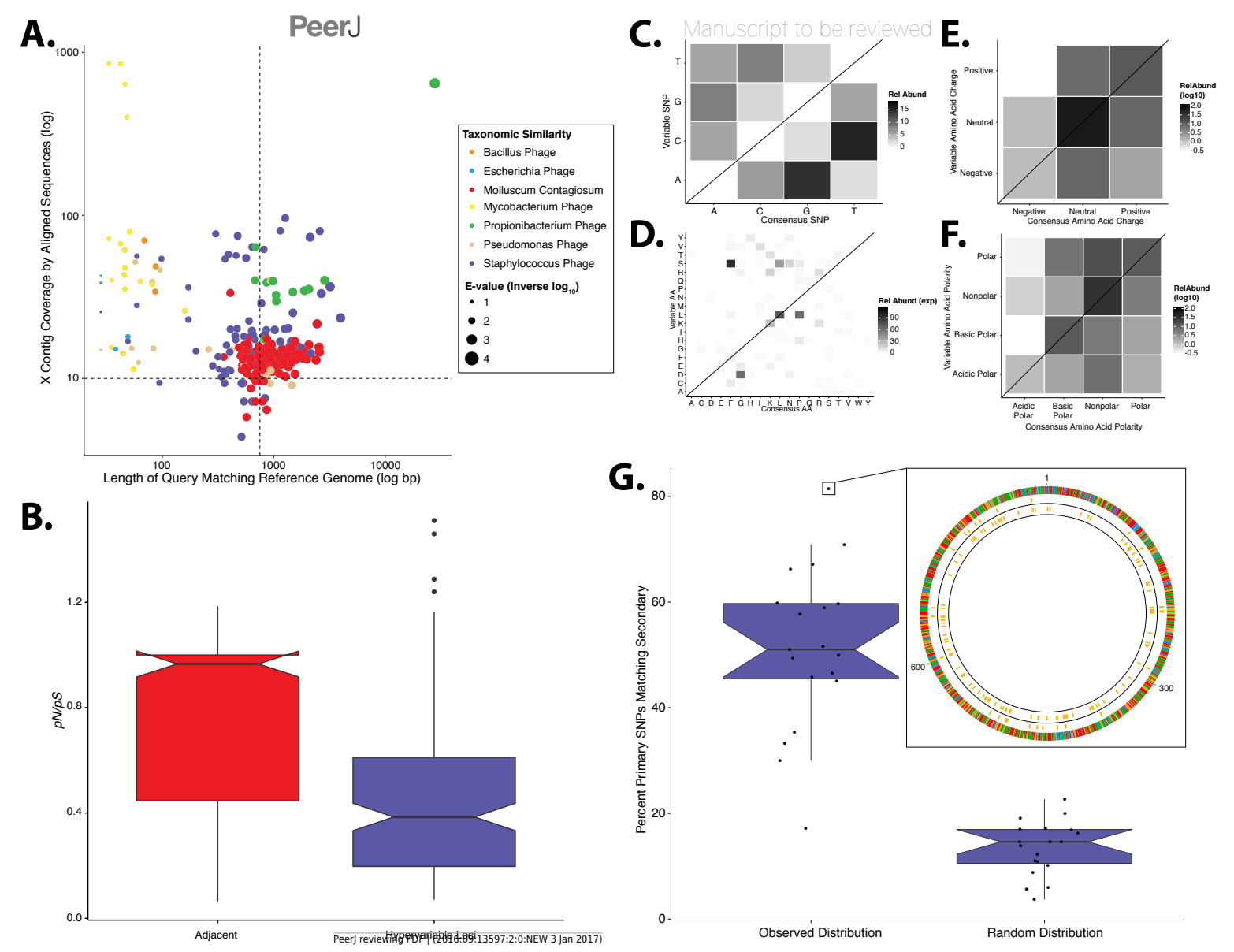